\title{
ANTIZIPATION INNERHALB DER WORTGRUPPE UND DIE SOG. BADALAPPOSITION IM ÄGYPTISCHEN
}

\author{
WOLFGANG SCHENKEL, 67 LU-Oggersheim, Schillerstraße 45, \\ West Germany
}

\section{Die Aufnahme Der sog. Badalapposition in die Beschreibung des Ägyptischen}

\section{DIE sog. Badalapposition, die J. Spiegel in der ZÄS 71 (1935) mit Blick auf die} arabische Nationalgrammatik zuerst in die Beschreibung des Ägyptischen eingeführt hat ${ }^{1}$, findet nach anfänglicher Zurückhaltung nun mehr und mehr Eingang in die Grammatiken des Ägyptischen. G. Lefebvre, der als erster an der Reihe gewesen wäre, von der Neuerung Gebrauch zu machen, hat es weder in der 1940 erschienenen 1. Auflage seiner Grammatik noch in der 2. Auflage von 1955 für nötig befunden, die Darstellung der Apposition über das hinaus zu erweitern, was vor Spiegels Aufsatz zur Standardausrüstung einer besseren Grammatik gehörte ${ }^{2}$. Auch A. H. Gardiner bleibt in der 2. Auflage seiner Grammatik (1950) und weiterhin in der 3. Auflage (1957) sachlich beim alten. Während aber Lefebvre den Terminus "Badal" nicht einmal erwähnt, etikettiert Gardiner nachträglich drei für uns von unseren eigenen Sprachen her auffällige appositive Konstruktionen mit diesem Terminus ${ }^{3}$, wobei ihm allerdings durch die Technik der Überarbeitung der Lapsus passiert, daß der zweite der drei Fälle überhaupt kein badal ist ${ }^{4}$, und der erste Fall nur im Sinne der arabischen Nationalgrammatik, nicht aber nach der Auffassung Spiegels dazugehört ${ }^{5}$.

Bis dahin stieß gerade der "Idealfall der Badal-Apposition"-wie W. Westendorf der heutigen Einschätzung entsprechend treffend wertet 6 - "mit Aufnahme des erklärenden Gliedes durch ein Suffix" noch auf keinerlei Gegenliebe. Spiegel scheidet eine "vollständige Form" der Badalapposition, mit Pronomen, vom Typ $A, B-f$ "A, genauer: sein B" von einer "verkürzten Form", ohne Pronomen, vom Typ $A, B$ "A, genauer: B" 8 und mißt eben dieser "vollständigen Form" die größere Bedeutung zu. Vermeldet wird die Form mit Pronomen dann aber 1955 in der Grammatik E. Edels ${ }^{9}$, der nun jedoch seinerseits bis auf die nicht ganz sicher zu beurteilende Form der Filiation vom

${ }^{1}$ J. Spiegel, "Zum Gebrauch der Apposition im Ägyptischen und Arabischen", ZÄS 71 (1935) (im folgenden: Apposition), 56-81. Auf das Arabische gehe ich nur so weit ein, wie es zur Klärung der Position Spiegels und der ägyptischen Grammatiken erforderlich ist.

2 G. Lefebvre, Grammaire de l'Egyptien classique (im folgenden: GEC), ${ }^{1} 1940$ und ${ }^{2} 1955, \$ 135$ f. Ältere Darstellungen: A. H. Gardiner, Egyptian Grammar (im folgenden: EG), ${ }^{1} 1927, \S 90 ;$ A. Erman, Ägyptische Grammatik (im folgenden: $\ddot{A} G$ ), ${ }^{4} 1928, \S 210$.

3 Gardiner, $\mathrm{EG}^{2-3}$, S. 68 , Anm. 4 a.

${ }^{4}$ S. Spiegel, Apposition, 57 (§ 1); 58-60 (§ 3). Vgl. H. L. Fleischer, "Ueber das Verhältniss und die Construction der Sach- und Stoffwörter im Arabischen", Kleinere Schriften, II 1, Leipzig 1888 (im folgenden: Verhältniss), 7. Es handelt sich um eine "gewöhnliche" Apposition oder, nach der Terminologie der arabischen Nationalgrammatik, um ein bajān.
Die Zusammenstellung Gardiners bleibt im übrigen, gemessen an Spiegels Ergebnissen, ebenso wie die der anderen zitierten Grammatiken unvollständig: es fehlen vor allem unter den "gewöhnlichen" Appositionen die Gruppe "Ganzes und seine Teile" (Spiegel, Apposition, 57 [§ 1]; 61-63 [\$5]) und die Gruppe "Form und Stoff," d. h. die Umkehrung der von Gardiner unter Punkt 1 verzeichneten und von ihm zur sog. Badalapposition gerechneten Konstruktion (Spiegel, Apposition, 57 [§ 1]; 60 f. [§ 4]).

5 Vgl. unten Anm. 20.

${ }^{8}$ W. Westendorf, Grammatik der medizinischen Texte, 1962 (im folgenden: GMT), § 139; auch die folgende Formulierung an dieser Stelle.

7 So dargestellt bei Westendorf, GMT § 139.

8 Spiegel, Apposition, 80 (§ 28).

o E. Edel, Altägyptische Grammatik, I, 1955 (im folgenden: AäG), §§ $306 \mathrm{f}$. 
Typ $B, z_{3} A$ "A, Sohn des B" alle Fälle ohne Pronomen ausscheidet und sie als "scheinbare Apposition" klassifiziert ${ }^{10}$. Westendorf hat in seiner Grammatik 1962 nur Beispiele ohne Pronomen zu behandeln; andere kommen in seinem Material nicht vor. Die Darstellung in Sander-Hansens Grammatik, $1963^{11}$, ist weder begrifflich noch sachlich sonderlich klar und hängt in $\S 643$ mit einer Fehleinstufung von Gardiner ab. Neben den Grammatiken ist F. Hintze anzuführen, der in seinen Untersuchungen zu Stil und Sprache neuägyptischer Erzählungen 1950 die Definitionen Spiegels kritisiert und aufgrund allgemein-sprachlicher Orientierung keinen scharfen Schnitt zwischen "gewöhnlicher" Apposition und sog. Badalapposition anzusetzen wünscht, der weiterhin der Konstruktion mit Pronomen einige Erwägungen widmet und sie ausdrücklich als sog. Badalapposition kennzeichnet ${ }^{12}$.

Gardiner und Lefebvre haben gut daran getan, den "Idealfall", mit Pronomen, nicht aufzunehmen; dieser Aufsatz hat nämlich das Ziel, diese Konstruktion aus den Appositionen wieder auszuscheiden.

\section{Badal UND bajān, "GEWÖHNLICHE" UND BADAL-APPOSITION}

Die arabische Nationalgrammatik kennt zwei Arten von Apposition: ein Substantiv, das ein anderes ersetzt, ist seine "Vertretung", badal; ein Substantiv dagegen, das ein anderes näher bestimmt, ist eine "erklärende Anfügung", "atf al-bajān ${ }^{13}$. Der bajān, die "Epexegese", ist bloße Nebenbestimmung eines Hauptbegriffs; der badal, das "Permutativ", hingegen umgekehrt der Hauptbegriff selbst ${ }^{14}$.

Spiegel bestimmt zwei Arten von Apposition so: "Die ['gewöhnliche'] Apposition ist die Hinzufügung eines Nomens zu einem anderen in dem Sinne, daß beide eine begriffliche Einheit bilden sollen. Dies setzt voraus, daß das durch das erste Nomen Bezeichnete identisch ist mit dem durch das apponierte Nomen Bezeichneten." ${ }^{15}$ Daneben besteht die Badal-Apposition, "bei der nur eine teilweise Identität zwischen dem Gegenstand des ersten und dem des apponierten Nomens besteht. Bei ihr enthält nämlich das apponierte Nomen den Gegenstand des ersten Nomens nicht ganz, sondern nur einen Bestandteil von ihm, bzw. etwas ihm Zugehöriges."16

10 Edel, AäG §§ 310-314.

11 C. E. Sander-Hansen, Ägyptische Grammatik, 1963, §§ 641; 643; 646.

12 F. Hintze, Untersuchungen zu Stil und Sprache neuägyptischer Erzählungen, I, 1950 (im folgenden: Untersuchungen), 117-119; $124 \mathrm{f}$.

13 Nach H. Reckendorf, Arabische Syntax, Heidelberg 1921 (im folgenden: Syntax), § 43, 1 .

${ }_{14}$ Nach Fleischer, Verhältniss, 9.

15 Spiegel, Apposition, 56 (§ 1).

16 Spiegel, Apposition, 56 (§ 1). Spiegel fährt fort: "Der Gegenstand des ersten Nomens ist dabei nur in einem Pronomen enthalten, das dem apponierten Nomen zugefügt wird. Der Gegenstand des apponierten Nomens selbst ist dagegen $\mathrm{n}$ i $\mathrm{c} \mathrm{h} \mathrm{t}$ identisch mit dem Gegenstande des ersten Nomens, sondern wird nur durch das zugefügte Pronomen als ihm zugehörig bestimmt. Die Zufügung des Pronomens ist hier also wesentlich, da nur durch es die notwendige Identitätsverbindung zwischen den beiden Nomina hergestellt wird." Die Behauptung, es sei ein Pronomen notwendig, und die andere, durch das Pronomen entstünde eine Identitätsverbindung (so auch noch einmal Apposition, 69), an der sich die Kritik Hintzes (Untersuchungen, 117 f.) entzündete, sehen wie

Schlacken einer anderen Redaktion des Aufsatzes aus, in der die Fälle ohne Pronomen noch nicht oder nicht genügend als vollwertige Konstruktion bedacht waren. Für das Ägyptische setzt Spiegel ja ausdrücklich die "verkürzte Form (ohne Pronomen)" an (Apposition, 69 f. [§ 12]; 80 [§ 28]). Die Rede von der "Identitätsverbindung" kann nach dem, was Spiegel sonst sagt (Apposition, 56 [§ 1]; 65 [§ 8]), besonders auch im Hinblick auf die Unterscheidung der Badalapposition von der "gewöhnlichen", deren Kennzeichen gerade die "Identität" sein soll, nur ein Lapsus sein; gemeint ist vielleicht: der sachliche Bezug oder die Relation zwischen den beiden Gegenständen.Hintze unterstellt im übrigen in seiner Kritik, da $\beta$ die "begriffliche Einheit", von der Spiegel im ersten Satz der Arbeit spricht, für die Apposition schlechthin, also auch für die Badalapposition gelten soll; dies ist nicht der Fall. Unter Apposition ist in diesem Satz ausschließlich die "gewöhnliche" Apposition zu verstehen, wie sich auch der ganze erste Absatz der Spiegelschen Arbeit nur auf diese bezieht; auf die Badalapposition kommt Spiegel erst im zweiten Absatz zu sprechen ("Im Arabischen gibt es d a n e b e n [von mir gesperrt] noch eine zweite Art der Apposition"). Hintzes Einwände sind sachlich 
Auf die anfechtbare Definition der "gewöhnlichen" Apposition einzugehen, ist hier nicht der Ort; sie ist lediglich hierher gesetzt, um das Bestimmungskriterium der Identität, mit dem Spiegel operiert, klarer vor Augen zu führen ${ }^{17}$. Es ist evident, daß Spiegels Abgrenzung der beiden Arten der Apposition nicht ganz die gleichen Sachverhalte trifft wie die Unterscheidung zwischen badal und bajān in der arabischen Nationalgrammatik. Die erste Untergruppe des badal, badal al-kull min al-kull "Vertretung des Ganzen für das Ganze" genannt ${ }^{18}$, schlägt Spiegel der "gewöhnlichen" Apposition zu; während die arabische Nationalgrammatik in ihrer Terminologie die Reihenfolge der Glieder berücksichtigt und z. B. die Verbindungen "Zählbares + Zahl", "Meßbares + Maß" als bajān von der umgekehrten Verbindung "Zahl + Zählbares", "Maß + Meßbares" als badal unterscheidet ${ }^{19}$, macht Spiegel gemäß seinem Kriterium der Identität oder Nicht-Identität hier keinen Unterschied und versteht einheitlich die "gewöhnliche" Apposition ${ }^{20}$. Gewiß erhebt sich hier die Frage, ob man im Ägyptischen angesichts der Strenge der geltenden Wortfolgeordnung gerade auf diese Unterschiede sorgfältiger achten müßte; ob man allerdings gerade mit badal und bajān sehr weit kommen wird, und ob überhaupt diese Unterscheidung der arabischen Nationalgrammatik sprachgerecht ist, ist durchaus zu bezweifeln. Eine Diskussion dieses Punktes führt indes zu sehr vom Thema $a b^{21}$. Festzuhalten ist, daß Spiegel durch seine neue Abgrenzung zwischen badal und "gewöhnlicher" Apposition die erste Art des badal aufgibt und dadurch zwangsläufig dazu gedrängt wird, die übrigen Arten des badal stärker zu betonen.

\section{Die vermeintliche Badalapposition mit Pronomen, eine WORTGGROPPE MIIT ANTIZIPATION}

Nach dem badal al-kull min al-kull sind zwei weitere der im ganzen vier Arten des badal der badal al-bacd min al-kull "die Vertretung des Teils für das Ganze" und der badal al-ištimāl "die Vertretung durch Mitumfassen" ${ }^{22}$. Beide sind durch das Vorhandensein eines Pronomens vor den anderen Arten ausgezeichnet. Spiegel nimmt sie als "vollständige Form" der Badalapposition auf und betrachtet sie geradezu als Idealform. Die Definition, die er Apposition, 56 (§ 1), gibt, ist auf diese Form zugeschnitten; vgl. oben Anm. 16. Es besteht kein Anlaß, die beiden Gruppen gemäß der arabischen Nationalgrammatik wieder nach inhaltlichen Kriterien auseinanderzunehmen; als Kennzeichen gilt, wie bei Spiegel, nur das Pronomen. Im übrigen beschränke ich mich ganz auf die Analyse der ägyptischen Konstruktionen; der Wurm indes sitzt bereits in der Behandlung des Arabischen durch die Nationalgrammatiker.

Die sog. Badalapposition hat nach der Darstellung Spiegels die Form Nomen ${ }_{1}+$ $\mathrm{Nomen}_{2}+$ Personalpronomen $^{23}$. Diese Form enthält folgende Einzelrelationen:

\footnotetext{
durch einzelne unzutreffende Formulierungen Spiegels gerechtfertigt, treffen aber doch ins Leere.

17 Was es mit der "Identität" auf sich hat, möchte ich an anderer Stelle aufgreifen.

18 Reckendorf, Syntax, $\S 43,1$.

10 Vgl. Fleischer, Verhältniss, 7 (Sache + Stoff: bajān); $8 \mathrm{f}$. (Zahl/Maß/Gewicht + Sache: badal); Reckendorf, Syntax, § 43, 1 ('ahūka Zaid: bajān; aber Zaid 'ahūka: badal).

20 Spiegel, Apposition, behandelt in den §§ 2-4 beide Konstruktionen zusammen.-Wenn Gardiner, EG $\S 90$, nachträglich von der 2. Auflage an die Verbindung Stoff + Sache als badal apostrophiert, so ist das wiederum (s. bereits oben Anm. 4), da or auf
}

Spiegel verweist, ein Lapsus; Spiegel bezeichnet diese Apposition als "gewöhnliche", nicht als Badalapposition. Die Bezeichnung Gardiners wäre allerdings im Sinne der arabischen Nationalgrammatik richtig!

21 Auf diesen Punkt möchte ich, soweit die Beschreibung der ägyptischen Verhältnisse betroffen ist, andernorts zurückkommen.

22 Reckendorf, Syntax, $\S 43,1$.

23 Spiegel, Apposition, 56 (§ 1), und die Belege ebd., 65-69 (\$§ 8-9). Die Formel $A, B-f$, die Westendorf, GMT § 139, dafür gibt, ist nicht ganz zutreffend, da das Personalpronomen nicht unbedingt ein Suffixpronomen sein muß; s. z. B. Spiegel, Apposition, 66 (erster hieroglyphischer Beleg). 
1. die Hinzufügung eines Nomens zu einem anderen Nomen $\left(\mathrm{Nomen}_{1}+\mathrm{Nomen}_{2}\right)$;

2. die Hinzufügung eines Personalpronomens zum zweiten Nomen $\left(\mathrm{Nomen}_{2}+\right.$ Personalpronomen); und

3. die Kongruenz des Personalpronomens mit dem ersten Nomen $\left(\right.$ Nomen $_{1} \cong$ Personalpronomen).

Es handelt sich also um eine Kombination mehrerer Relationen. Man kann diese Zusammensetzung trotzdem in der Grammatik als e i n e Konstruktion vorstellen, wenn es die Teilrelationen sonst nirgends gibt. Es gibt sie jedoch im Ägyptischen. Die Kongruenz ist außerhalb dieser Konstruktion gang und gäbe. Die unter Punkt 2 begriffenen Relationen sind samt und sonders anderweitig geläufig, z. B., der häufigste Fall, der "Genitiv" (d. i. hier: Substantiv + Personalsuffix); zu anderen Relationen, die hierher gehören, gleich. Somit bleibt allein das Verhältnis des Punktes 1 unaufgelöst. Die Frage ist schließlich diese: was steckt in der Gesamtkonstruktion abzüglich der Kongruenz und der zwischen $\mathrm{Nomen}_{2}$ und Personalpronomen geltenden Relation noch darin?

Knüpfen wir an Spiegel an, der sich auch zur Relation zwischen $\mathrm{Nomen}_{1}$ und $\mathrm{Nomen}_{2}$ äußert! Zu einem Beleg mit Suffixpronomen vermerkt er: "Die appositionelle Verbindung entspricht hier einer Genetivverbindung"' ${ }^{24}$, weitet diese Relation aber später unbedacht auf die Badalapposition allgemein aus ${ }^{25}$. Wie aufgrund der Kongruenz des Personalpronomens mit dem ersten Nomen gar nicht anders zu erwarten, steht das erste Nomen zum zweiten Nomen im gleichen semantischen Verhältnis ${ }^{26}$ wie das Personalpronomen zum zweiten Nomen, nur ist in beiden Fällen die Richtung dieses Verhältnisses umgekehrt (Nomen $\rightarrow$ Nomen $_{2} \leftarrow$ Personalpronomen); dabei ist nicht nur eine "Genitiv"Relation möglich, wie man leicht wegen des statistischen Utbergewichts des "Genitivs" glauben könnte. Spiegel hat in seinen meist aus dem Neuen Reich stammenden, aber z. T. alten Belegen ${ }^{27}$ folgende Funktionen des Personalpronomens (und damit indirekt auch des ersten Nomens) zum zweiten Nomen notiert:

1. sog. logisches Subjekt (Suffixpronomen), z. B. $m_{-}{ }^{c} j$.f jrj.tn.f "bei ihm, d. h.: (bei) dem, was er getan hatte";

2. sog. logisches Objekt (enklitisches Pronomen), z. B. grg $\underline{d} d s w$ "die Lüge, d. h.: der, der sie sagt";

3. Satellit einer Präposition (Suffixpronomen) ${ }^{28}$, z. B. (n) p.t (n) ntr.w ntr.wt jmj.w.s "(für) den Himmel, d. h.: (für) die Götter und Göttinnen in ihm" "29;

4. sog. Genitiv (Suffixpronomen), z. B. ntr.w nb.w cj.wj.sn "alle Götter, genauer: ihre Arme".

Setzt man statt "logisches Subjekt" und "logisches Objekt" schlicht "Subjekt" und "Objekt" ein ${ }^{30}$, so klingt eine ganz geläufige Konstruktion an, nämlich die A n t i z i -

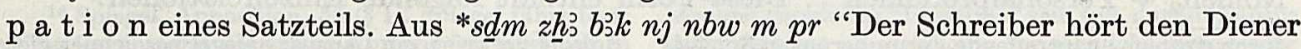

24 Spiegel, Apposition, 57, Anm. 1; vgl. 73 f. (§ 16).

25 Spiegel, Apposition, 69, Anm. 8; vgl. auch 73 (§ 16, Anfang).

26 Auf eine klarere Bezeichnung möchte ich mich hier nicht festlegen; für den Fortgang der Untersuchung ist eine weitere Klärung überflüssig.

27 Die Frage, inwieweit zwischen den verschiedenen Sprachstufen in der Verwendungsbreite der Konstruktion Unterschiede bestehen, muß ich offen lassen.

$28 \mathrm{Zu}$ dieser "präpositionalen Relation", die nicht mit dem sog. direkten Genitiv einfach gleichgesetzt werden darf, vgl. W. Schenkel, "Die mittelägyptischen Nisben als Nuklei in präpositionaler, limitierender und Genitiv-Relation", Chronique d'Egypte, XLI/81 (1966), 50-59.

29 Zur Wiederholung der Präposition vgl. unten, Abschnitt IV (b.)

${ }^{30}$ In anderem Zusammenhang werde ich zeigen, daß diese Ersetzung legitim ist, und darlegen, auf welchem Weg man die traditionellen Schwierigkeiten, die zu den "logischen" Satzteilen führen, überhaupt umgehen kann. 
des Herrn im Haus" kann man durch Antizipation eines Satzteils folgende Sätze ableiten:

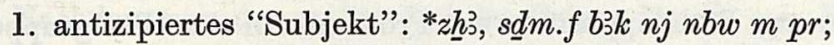

2. antizipiertes "Objekt": *bjk nj nbw, sd $m$ sw $z \underline{h}$ ’ $m$ pr;

3. antizipierter Satellit der Präposition: * $p r, s \underline{d m} z \underline{\jmath}$ ว $b_{3}^{\jmath} k$ nj $n b w$ jm (.f);

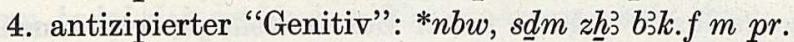

Die anderen zwei Kennzeichen der sog. Badalapposition mit Pronomen, das Vorhandensein eines Personalpronomens und die Kongruenz des Personalpronomens mit dem ersten Nomen, begegnen in den Sätzen mit antizipiertem Satzteil in vergleichbarer Weise. Der wesentliche Unterschied zwischen den beiden Fällen ist der, daß der Rahmen, innerhalb dessen ein Nomen antizipiert wird, einmal der Satz ist, das andere Mal eine Wortgrup $\mathrm{e}^{31}$.

Die sog. Badalapposition mit Pronomen ist somit als Wortgruppe mit Antizipation eines Gliedes aufzulösen. Diese Lösung hat den Vorteil, daß man keinerlei neue Kategorie in die Grammatik für diese Spezialkonstruktion mit Pronomen einführen muß, und zweitens den entschiedenen Vorzug, daß die anderen, vorher in die komplexe Konstruktion einverleibten, aber auch sonst in der Grammatik geläufigen Teilrelationen (Kongruenz; eine Funktion wie z. B. "Objekt" usw.), aus der Zusammensetzung herausgelöst sind. Spiegel hat bereits den Vergleich mit den Sätzen mit Antizipation eines Satzteils in seinen abschließenden Überlegungen zum Wert der sog. Badalapposition schön ausgeführt ${ }^{32}$, allerdings keine Konsequenzen daraus gezogen ${ }^{33}$.

\section{Einzelne Bemerkungen zo den Wortgruppen mit Antizipation}

(a) Wie im Satz kann auch in der Wortgruppe ein Personalpronomen antizipiert werden; z. B. $m h f^{c} . f h m . f$ "in seiner, d. h. seiner Majestät Faust" "34. Vgl. die Antizipation $n t f s \underline{d} m \cdot f^{35}$.

(b) Bei der Antizipation in einer Wortgruppe, die von einer Präposition abhängt, kann, was bei der Antizipation im Satz kaum vorkommt, die Präposition einmal vor dem antizipierten Nomen und ein zweites Mal vor dem Rest stehen (z. B.: jm.j m nn $\underline{d} d . n . j$ "über mich, genauer: über das, was ich sagte" ${ }^{36}$ ), oder aber nur einmal, und zwar vor dem antizipierten Nomen (z. B. $m$ - $^{c} j . f j$ jrj.tn. $f$ "bei ihm, d. h.: bei dem, was er getan hatte" $\left.{ }^{37}\right)$. Die Wiederholung der Präposition ist nicht verwunderlich, da auf diese Weise die Einbindung der unselbständigen, in antizipiertes Nomen und Rest aufgeteilten Wortgruppe als Satzglied in den Satz verstärkt wird.

$31 \mathrm{Zu}$ "Wortgruppe" in anderem Zusammenhang weitere Erläuterungen.

32 Spiegel, Apposition, $73 \mathrm{f}$. (§ 16).

${ }^{33}$ Anhangsweise noch eine Bemerkung zu einem Punkt, der für die Beweisführung Spiegels einmal von hoher Bedeutung war. Spiegel sieht sich wegen der Einschätzung der Appositionen als Korrelaten von "Identitätssätzen" gezwungen, die den sog. Badalappositionen entsprechende Satzform nachzuweisen. Seinen Belegen (Apposition, $72 \mathrm{f}$. [§ 15] fehlt nun allerdings gerade ein entscheidendes Kennzeichen der sog. Badalapposition, die Kongruenz zwischen dem Personalpronomen und dem Nomen; vgl. 3h.w.s $m$ ntr "Ihr Wesen ist (das eines Gottes)/ ein Gott". Bestünde die Parallelität mit.der sog. Badalapposition, so müßte das Personalsuffix mit ntr kongruieren; man hätte dann z. B. *"Der Gott ist sein (eigenes!) Wesen". Solche Sätze sehen wohl ägyptisch aus, so daß also, legt man diese zugrunde, die für Spiegel unabdingbare Demonstration doch noch möglich wäre. Da jedoch von einem Identitätssatz nicht unbedingt auf die Existenz einer entsprechenden Apposition geschlossen werden mußauch Spiegel schließt ja nur umgekehrt von der Apposition auf den "Identitätssatz"-, ist dieser Punkt für die Anerkennung oder Nicht-Anerkennung der sog. Badalapposition als Apposition unerheblich.

34 Mehrere Beispiele bei Spiegel, Apposition, §§ 8-9.

${ }^{35}$ Die nicht mit der futurischen Konstruktion $n t f$ $s d m$. $f$ zu verwechseln ist, vgl. W. Schenkel, "Beiträge zur mittelägyptischen Syntax", II, ZÄS 88 (1963), 121.

36 Mehrere Beispiele bei Spiegel, Apposition, §§ 8-9. 37 Mehrere Beispiele bei Spiegel, Apposition, §§ 8-9. 
(c) Die von Spiegel, Apposition, §§ 13 f., besprochenen und mit Hilfe der sog. Badalapposition erklärten Partizipialkonstruktionen und Relativsätze, sind durchaus richtig verstanden. Die Umdeutung der sog. Badalapposition mit Pronomen in eine Wortgruppe mit Antizipation verlangt nur geringfügige Retuschen; ich möchte diesen Punkt in günstigerem Zusammenhang anderwärts noch einmal aufgreifen.

(d) Die Verbindungen $\underline{d s . f}$ usw. "selbst" ${ }^{38}$, deren erster Bestandteil, $\underline{d s}$, selten mehr als selbständiges Substantiv belegt ist ${ }^{39}$, sind nach ihrer Erstarrung in die Funktionswortart der Adjektive ${ }^{40}$ einzureihen; ihre Kongruenz ist vergleichbar mit der des

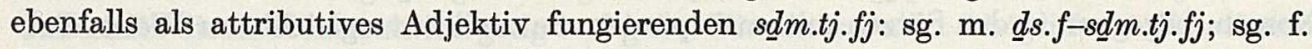
$\underline{d} s . s-s \underline{d} m . t j . s j ;$ pl. $\underline{d} s . s n-s \underline{d} m . t j . s n$. Bei erstarrtem $\underline{d} s . f$ usw. von Antizipation zu reden, geht nicht an, weil der Wortgruppe mit Antizipation eine solche ohne Antizipation entsprechen sollte, in der das Suffixpronomen durch einen "Genitiv" ersetzt wäre; von Relikten abgesehen, ist eine solche Verbindung im Ägyptischen, zum mindesten aber im sog. Mittelägyptischen, nicht mehr möglich.

\section{AFFEKT- UND INTELlektualkonstruktion}

Die Antizipation, eines der wenigen Mittel des Ägyptischen, von der starren Wortfolgeordnung abzuweichen, in ihrem Wert und ihrem Wirken zu beschreiben, übersteigt den Rahmen dieser Untersuchung. So weit die Antizipation in der Wortgruppe, d. h. die sog. Badalapposition mit Pronomen, betroffen ist, haben Spiegel und Hintze bereits wichtiges gesagt"1. Sie gründet sich auf dem "Wunsche nach stärkerer. Hervorhebung des Ganzen neben dem Bestandteil" ${ }_{42}$, während die andere Form der Antizipation, die im Satz, "die Betonung des Ganzen gegenüber anderen Ganzen bezweckt" 43 .

Hintze hat nun allerdings den affektischen Charakter der sog. Badalapposition mit Pronomen-das einzige Beispiel der Badalapposition, das er bespricht, ist von diesem Typ-überschätzt, die Badalapposition insgesamt als "sprachliche Fehlleistung infolge Affekts, starker Mitbeteiligung, oder geringer Rücksichtnahme auf den Hörer" eingestuft und sie zu den Anakoluthen gestellt ${ }^{44}$. Ohne Zweifel gibt es zwischen reiner Intellektualkonstruktion und extremer, kaum mehr verständlicher Affektkonstruktion eine breite Skala von Zwischenwerten. So gewiß die Antizipation und auch speziell die Antizipation in Wortgruppen gegenüber den Konstruktionen ohne Antizipation ausdrucksstärker, affektisch ist, so fern steht sie doch den Anakoluthen. Dazu ist dieses Mittel im Ägyptischen schon zu geläufig, in zu hohem Grad grammatikalisiert. Ich möchte meinen, daß sie der Intellektualkonstruktion näher steht als dem Anakoluth. Zutreffen kann dagegen Hintzes Einstufung als Anakoluth, oder, wie die arabische Nationalgrammatik sagt, als badal al-galat, auf einen Teil der noch verbleibenden Fälle der sog. Badalapposition, ohne Pronomen, die er allerdings nicht zitiert.

$38 \mathrm{Vgl}$. Spiegel, Apposition, 69 (§ 10).

39 Erman-Grapow, Wörterbuch, V, 607, 4-5.

${ }^{40} \mathrm{Zu}$ "Adjektiv" Näheres an anderer Stelle.

$₫ 1$ Spiegel, Apposition, $73 \mathrm{f}$. (§ 16), über den Unterschied zwischen "Genitiv", antizipiertem "Genitiv" in der Wortgruppe und Antizipation des "Genitivs" im Rahmen des Satzes; Hintze, Untersuchungen, $124 \mathrm{f}$., über den antizipierten "Genitiv" als affektische Konstruktion.

42 Spiegel, Apposition, 73; auch das folgende Zitat an dieser Stelle.

43 Für die Antizipation in der Wortgruppe ist im übrigen die gute Konstruktion deutscher Dialekte vom Typ "dem Tischler sein Haus", die K. Sethe, ZÄS 49 (1911), 99, in die Diskussion brachte, Spiegel aber Apposition, 74, Anm. 1, ausscheiden zu können meinte, doch ein recht guter Vergleich. Gegenüber der Konstruktion vom Typ "das Haus von dem Tischler", deren Existenz Spiegel übersieht, legt sie-bei gleicher Betonung auf dem ersten Substantivgrößeres Gewicht auf den "Genitiv". Man vergleiche Konstruktionen vom Typ "Ihre Mutter" neben dem Typ "Ihnen Ihre Mutter"; die zweite Konstruktion legt gegenüber der ersten unverkennbar stärkeres Gewicht auf den "Genitiv".

44 Hintze, Untersuchungen, $124 \mathrm{f}$. 


\section{Die sog. Badalapposition ohne Pronomen}

Nachdem nun der größte Teil der Belege für die sog. Badalapposition ausgeschieden und anders in die Grammatik eingeordnet ist, geht es nicht mehr an, den kleineren Belegrest weiterhin unter der Fahne der "Badalapposition" segeln zu lassen. Der Abstand vom badal der arabischen Nationalgrammatik ist zu groß, als daß eine Übertragung gerechtfertigt wäre, selbst wenn der Rest sich auf einen gemeinsamen Nenner bringen läßt. Aber auch dies ist schwerlich möglich. Was tut man mit dem Rest? Was bleibt überhaupt noch übrig?

Spiegel hat bereits in einem wichtigen Punkt, bei den Zahl-, Maß- und Stoffangaben, die Grenze zwischen der Badalapposition und der "gewöhnlichen" Apposition zugunsten der "gewöhnlichen" verschoben ${ }^{45}$. Man mag ihm recht geben. Allerdings ist nun die Frage nach dem Sinn der verschiedenen Reihenfolge ganz neu im Rahmen der "gewöhnlichen" Apposition aufzunehmen.

Es könnten weiter die Entsprechungen der 4. und letzten Art des arabischen badal, des badal al-galaț "der Vertretung wegen Irrtums" ${ }^{46}$, auftreten. Es handelt sich hier nicht um eine normale grammatische Konstruktion, sondern um ein Anakoluth. Es dürfte nicht ganz einfach sein, solche Anakoluthe in ägyptischen Texten einwandfrei nachzuweisen, da man immer mit Schreibfehlern rechnen muß. Wären Anakoluthe Stilmittel, so müßten sie sich durch metrische Textanalyse sichern lassen, vorausgesetzt, daß es das metrische Netz mit der entsprechenden Maschenweite gibt.

Die Filiation ohne Pronomen vom Typ $A z_{\supset} B$ "A's Sohn B" ${ }^{3}$ ist, wie man das Verhältnis zur älteren Konstruktion vom Typ $A$ z.f $B$ auch sehen mag, eine erstarrte Formel, die als solche auch lebensfähig ist, wenn sie sich nicht nach den Regeln der zeitgenössischen Syntax auflösen läßt.

Von den übrigen sog. Badalappositionen ohne Pronomen, die Spiegel zusammengestellt hat ${ }^{48}$, ist die letzte eine ganz "gewöhnliche" Apposition; der Ansatz als Badalapposition beruht auf falscher Analyse ${ }^{49}$. Den vorausgehenden Belegen ist gemeinsam, daß sich das durch den apponierten Ausdruck Bezeichnete $\mathrm{r}$ ä $\mathrm{u} \mathrm{m} \mathrm{l}$ i c h innerhalb des durch den ersten Ausdruck Bezeichneten befindet. Vergleichbar ist der Stil unserer Adressen wie "Heidelberg, Akademiestraße 5". Edel erklärt den geläufigsten Typ $T$ ว-wr(r) วbd $\underline{\jmath} w$ "Abydos im Thinitischen Gau" 50 zu einer scheinbaren Apposition ${ }^{51}$. Die Sprache wird dabei zu sehr auf eine bestimmte Norm festgelegt. Selbst wenn die Konstruktion nur amtlich-papieren ist, ist sie doch offensichtlich sprachrichtig.

Mit diesen Bemerkungen lasse ich es bewenden. Die letzten Beispiele rechtfertigen, isoliert für sich betrachtet, noch nicht den Ansatz einer eigenen Art von Apposition. Auch sie zeigen jedoch, daß das große Reservoir der Appositionen, in das nun alle Belege

45 S. oben Abschnitt 2.

46 Reckendorf, Syntax, $\S 43,1$.

47 Spiegel, Apposition, 69 (§ 11); weiter: K. Sethe, ZÄS 49 (1911), 95-97; 99; Edel, AäG § 307.

48 Spiegel, Apposition, $69 \mathrm{f}$. (\$ 12).

49 Spiegel versteht so, als ob $s p r$. $s n w^{c} j . w r w^{c} j . w$ dastünde, und der ganze Ausdruck $w^{c} j . w \quad r \quad w^{c} j . w$ Apposition wäre ( $\mathrm{Zu}$ solchen Konstruktionen s. H. Paul, Prinzipien der Sprachgeschichte, ${ }^{5} 1920\left[{ }^{6} 1960\right]$, 155 f. [\$ 107]; Paul spricht von "partitiver Apposition", vgl. Register s. v. Apposition). In Wirklichkeit steht $s p r . f w^{c} j . w r w^{c} j . w$ da, und nur das erste $w^{c} j . w$ ist Apposition, nämlich zu .f. Die Kompliziertheit des Belegs hat eine andere Ursache, daß nämlich in einer Konstruktion spr $X r Y$ das Wort $X$ einerseits, um den Bezug auf ein vorausgehendes Wort zu kennzeichnen, ein Personalpronomen $(. f)$ sein muß, andererseits $X$ und $Y$ mit korrespondierendem $w^{c} j . w-w^{c} j . w$ in eine Antithese gesetzt werden.

50 Es kommit auch der Typ $3 b d \underline{d} w$ T3-wr $(r)$ vor, vgl. Lefebvre, GEC ${ }^{2}$ \& 136. Dazu vgl. das anderwärts geläufige "Akademiestraße 5, Heidelberg"; andere Beurteilung: H. Kees, ZÅS 73 (1937), $88 \mathrm{f}$. 51 Edel, AäG § 314. 
hineingeschüttet wurden, nicht lauter völlig homogene Fälle enthält, daß vielmehr innerhalb der Apposition eine Schichtung besteht. Diese verschiedenen Schichten herauszuarbeiten wäre wohl eine eigene Untersuchung wert. Als unbrauchbar erwies sich zunächst nur einmal die Aufteilung in "gewöhnliche" Apposition und Badalapposition. Die nun noch bestehenden Unterschiede zwischen den verschiedenen Schichten sind subtiler, als es bei denen zwischen den zwei großen, von Spiegel geschiedenen Gruppen der Fall war, so daß man jetzt vor allem einmal auf die Zusammengehörigkeit aller appositiven Verhältnisse zu einer einzigen grammatischen Kategorie Gewicht legen muß, wie sie auch Hintze auf dem Hintergrund einer allgemein-sprachlichen Orientierung richtig schien ${ }^{52}$.

52 Hintze, Untersuchungen, $118 \mathrm{f}$. 